\title{
Why do Patients Still Catch Hospital Infections Despite the Practice of Infection Prevention and Control Programs?
}

\section{Huang Wei Ling*}

MD Infectious Disease Specialist (ID Specialist), General Practitioner, Nutrition Doctor, Acupuncturist, Pain Management, Medical Acupuncture and Pain Management Clinic, Franca, Sao Paulo, Brazil

*Corresponding Author: Huang Wei Ling, MD Infectious Disease Specialist (ID Specialist), General Practitioner, Nutrition Doctor, Acupuncturist, Pain Management, Medical Acupuncture and Pain Management Clinic, Franca, Sao Paulo, Brazil.

E-Mail: weilingmg@gmail.com

Received: February 06, 2018; Published: March 20, 2018

DOI: 10.31080/ASMI.2018.01.0036

\begin{abstract}
Statement of the Problem: Very few publications provide sound scientific data used to determine which components are essential for Infection Prevention and Control (IPC) programs in terms of effectiveness in reducing the risk of infection. In recent years, a range of regional best practice or policy principles have been developed that address what could be considered as core components of IPC programs. However, there remains a major gap in relation to the availability of international best practice principles for core components of IPC programs.

Purpose of this Study: The purpose of this study is to show why patients still catch hospital infections despite IPC programs. A better understanding of a variety of theories is needed that could explain the physiopathology of diverse diseases described in the medical past history, which are usually disregarded clinically today. A broader view seems to show the necessity of seeing the patient as a whole; not only focusing on the disease in the prevention of these hospital infections.

Methodology: The methodology used was a review of these theories such as those presented by Hippocrates ("Natural forces within us are the true healers of disease"), as well as others from oriental medicine, which explain that diseases originate from three factors: external (exposure to cold, heat, humidity, wind and dryness), internal (emotional) and dietary.

Findings: Having a broader view of the patient as a whole (Yin, Yang, Qi, Blood energy and Heat retention), we can understand better the formation of hospital infection which is a systemic energy reaction of our body undergoing normal hospital treatment.

Conclusion: To better understand why a patient is still catching hospital infections, despite these IPC programs, we need to broaden our view observing all emotional, environmental and dietary factors, as well as studying his energy situation at the moment of admittance, finally identifying his susceptibility to the risk of hospital infections.
\end{abstract}

Keywords: Hospital Infection; Prevention; Control Programs; Energy; Traditional Chinese Medicine; Acupuncture; Galen; Hippocrates

\section{Introduction}

Health care-associated infections (HAIs) are the most common complication seen in hospitalized patients. HAIs increase morbidity, mortality, costs, and length of stay even after adjustment for underlying illness [1]. The term nosocomial infection encompasses a narrower spectrum. Nosocomial infections are HAIs acquired in an acute-care setting that were neither present nor incubating at the time of admission [1]. Based on data for 2003,5 to $10 \%$ of patients admitted to acute-care hospitals or approximately 2 million patients per year in the United States acquire a nosocomial infection. At least 90,000 deaths per year are a result, making nosocomial infections the fifth leading cause of death in acute-care hospitals [1]. These infections are estimated to add an extra \$4.5 billion to \$5.7 billion per year to the cost of patient care [1]. Roughly $25 \%$ of nosocomial infections occur in intensive care units (ICUs), which have been estimated to increase ICU length of stay by 4.3 to 15.6 days and account for more than $20 \%$ of total ICU costs. The Centers for Disease Control and Prevention (CDC) defines health care-associated infections (HAIs) as infections acquired while in the health care setting (e.g. inpatient hospital admission, haemodialysis unit, or same-day surgery), with a lack of evidence that the infection was present or incubating at the time of entry into the health care setting. These definitions need to respond to a changing medical environment [1].

In 1980, the Study on the Efficacy of Nosocomial Infection Control (SENIC) demonstrated that surveillance for nosocomial infections and infection control practices that included trained professionals could prevent HAIs [1]. As a result, an important role developed for hospital epidemiologists and infection control practitioners [1]. As medical care has become more complex, an- 
timicrobial resistance and HAIs have increased, as have their attributable morbidity and mortality [1]. Additionally, HAIs increase hospital lengths of stay and health care expenditures [1]. In response to patient risks and growing costs, in 2008 the Centers for Medicare and Medicaid Services (CMS) implemented a strategy of withholding reimbursement for certain HAIs such as catheter-associated urinary tract infections (CA-UTIs) and central line-associated bloodstream infections (CLABSIs) [1]. Now more than ever, institution-specific surveillance driven by hospital epidemiologists and infection preventionists (IPs) is needed in order to enact early detection and preventive strategies to curtail HAIs [1].

The theory-practice gap has always existed [2]. This gap is often cited as a culmination of theory being idealistic and impractical, even if practical and beneficial, is often ignored. Most of the evidence relating to the non-integration of theory and practice assumes that environmental factors are responsible and will affect learning and practice outcomes, hence the gap [2].

Although infectious agents have existed since long before humanity, and despite the rapid advances characterizing the previous century in terms of the recognition and treatment of the diseases induced by these agents, infection remains a major cause of human morbidity and mortality, and an ever-present threat. The latter fact has often led specialists to look back in order to either underline a specific pathogen's importance to humanity or to seek ideas regarding pathophysiology and treatment. In looking into the past for options for the future, the heritage of Hippocrates is of great significance [3].

Exploring the past will almost definitely provide a more progressive way of thinking, and this is nowhere more pronounced than in the field of infectious diseases - the Hippocratic heritage and the various ways it is still perceived today [3]

Traditional Chinese Medicine (TCM) believes that man is an organic unity in which the component parts of the human body are inseparable in structure, kind of idea, unity of the internal and external environments and the unity of the human body itself, is known as the concept of "holism", which is carried out in almost every aspect of TCM, such as physiology, pathology, diagnostic methods, Syndrome identification and treatment [4].

The researcher has much both practical and theoretical experience in the area of this study. Be it, by the control of hospital infections - obtained in the daily contact with patients who have suffered from this kind of infection, be it by her own observations and ideas which came from a diversity of studies, a diversity of understandings which lead to the way the subject is presented today. Thus, new concepts, new points of view are brought in the attempt to improve such a serious problem in our hospitals worldwide.

\section{Purpose}

The purpose of this study is to show why patients still catch hospital infections despite IPC programs. A better understanding of a variety of theories is needed that could explain the physiopathology of diverse diseases described in the medical past history, which are usually disregarded clinically today.

\section{Methodology}

The methodology used was a review of these theories such as those presented by Hippocrates ("Natural forces within us are the true healers of disease"), as well as others from oriental medicine, which explain that diseases originate from three factors: external (exposure to cold, heat, humidity, wind and dryness), internal (emotional) and dietary.

Before describing this study, it is important to say that not everything we capture with our eyes represents what we actually see. Depending on the angle or framework we focus an object, what we see changes shape or appearance. This study will bring a different understanding regarding the identification of what is referred to as infection in traditional Western medicine.

An illustrative example of what can happen to the human body, any redness, bronchial or other purulent secretion, fever, urinary pain, etc... may be an indication of infection. Our patients' signs and symptoms can be seen differently depending on the perspective employed. As a consequence, there are other points of view, like the one within the millenary teachings of Traditional Chinese Med icine (TCM). Any symptoms of physical, emotional or psychological illness is caused by the imbalance of what is named as the internal energies ( $Q i$ in Chinese)

\section{Qi in Chinese Medicine}

The term energy in Chinese medicine or $Q i$ is characterized as the image of steam rising from a pot of rice cooking on the stove (Figure 1). That steam represents Qi... (Figure 1). Qi is also called life energy, life force, prana, and bio-energy as well as many other names. For thousands of years, this energy has been known to every culture in almost all parts of the world. Some of them explored it and some did not. Those who did discovered a whole new world [5]. Our energy is similar to electricity in a battery, or combustible gas in a cylinder or even gasoline at a gas station.

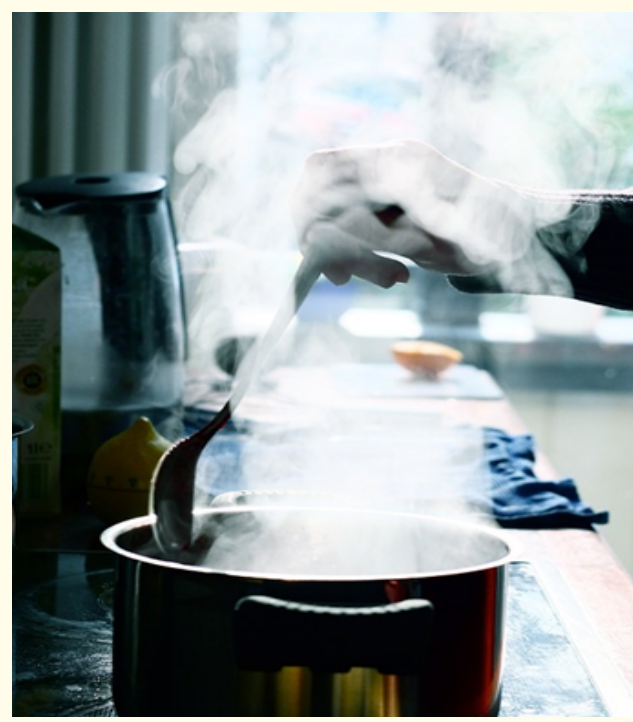

Figure 1 


\section{Phases of the disease}

Before a disease is diagnosed at the laboratory, through radiological level or by complementary tests, there has already been an alteration in energy levels, the patients have symptoms but the tests are still normal (Table 1). There are various phases where disease progresses in a healthy body. During phases 1 to 3 , only a slowing of the organ functions occurs because of energy imbalances, and the patient has and complains of symptoms, but the results of laboratory exams are normal (Table 1). At phase 4 the laboratory exams show some alterations, and the disease is still curable, and at phase 5 , the exams are very altered, and the cellular damage is irreversible and the disease incurable (Table 1).

\begin{tabular}{|l|c|c|c|c|}
\hline \multicolumn{5}{|c|}{ Progression of Health to Disease } \\
\hline Phase 1 & $\begin{array}{c}\text { Organ } \\
\text { Slowing Down of the } \\
\text { organ functions }\end{array}$ & Normal & Energy Reserve & Symptom \\
\hline Phase 2 & $\begin{array}{c}\text { Slowing Down of organ } \\
\text { functions }\end{array}$ & Normal & $\begin{array}{c}\text { Consumption of internal } \\
\text { energy reserves }\end{array}$ & With symptoms in other organ \\
\hline Phase 3 & $\begin{array}{c}\text { Slowing Down of organ } \\
\text { functions }\end{array}$ & Normal & $\begin{array}{c}\text { Consumption of external } \\
\text { energy reserves }\end{array}$ & With symptom in the same organ \\
\hline Phase 4 & $\begin{array}{c}\text { Reversible Cellular Le- } \\
\text { sion }\end{array}$ & Little alteration & $\begin{array}{c}\text { Consumption of blood } \\
\text { reserves }\end{array}$ & Curable disease \\
\hline Phase 5 & $\begin{array}{c}\text { Irreversible Cellular } \\
\text { Lesion }\end{array}$ & Excessive alteration & Metabolic exhaustion & Incurable disease \\
\hline
\end{tabular}

Table 1: Progression of Health to Disease.

Meaning of infectious symptom in Traditional Chinese Medicine

In the oriental tradition, when diseases occur, it is a sign that there is an imbalance of this energy, generally caused by emotional, dietary or external factors, or a combination of them [4]. These imbalances can manifest in many ways. Here are some examples: in the case of discharge of pus, this is considered to be the retention of Humidity and Heat. The Humidity could occur because of the deficiency of energy in the Spleen - which is responsible for the absorption of nutrients and maintaining the blood in the vessels. The Heat retention could be the consequence when external pathogenic factors like cold internalize in the body and make the secretions transmute from colorless to yellowish [4]. One of the causes of Spleen deficiency is found in the diet with the use of dairy products, sweets, raw food, and cold drinks [4]. Another cause is found among those who are constantly, and overly worried [4]. In the case of redness in the skin or in the surgical incision, for example, this is considered to be the formation of excessive Heat inside the body, which can also come from an inadequate diet, deficiency of energy or emotional issues such as anger [4]. As for an inappropriate diet, this includes the use of fried food, chocolate, eggs, honey, alcoholic drinks, coconut, pepper and dairy products especially when cheese is melted

\section{Yin and Yang}

The symmetrical arrangement of the dark Yin and the light Yang is not static (Figure 2). It is a rotational symmetry that eloquently suggests a continuous cyclic movement: the Yang cyclically returns to its beginning, the Yin reaches its apogee and yields its place to the Yang $[4,7]$. The two points in the diagram symbolize the idea that every time each force reaches its extreme point, it manifests within itself the seed of its opposite. The Chinese wisemen represented this complement of opposites by the archetypal pair, Yin and Yang, considering their dynamic interaction as the essence of all natural phenomena and all human situations [4,7] (Figure 2). Opposites are complementary [4]. To be healthy, we aim for a state of equilibrium between the Yin and Yang energies [4]. If there are imbalances between these two energies, the appearance of symptoms occurs, and if not properly treated, consolidates the disease in the future, being diagnosed at the laboratory or radiological level [4].

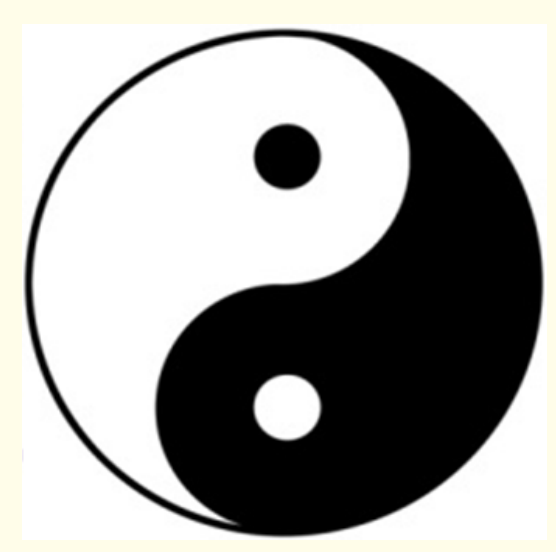

Figure 2

How to do the diagnose of energy deficiency

To diagnose the energy deficiencies, patients are asked if they have a daily bowel movement and if not, it could mean Blood deficiency. If a patient has excessive sweating during the day, this is a sign of $Q i$ deficiency. If he feels more cold than hot especially in his extremities such as cold feet, it is a sign of Yang deficiency. When the patient feels hot, especially in his extremities, it can be Yin deficiency. The diagnosis of Heat retention is based when we ask the patient if he has dry mouth, bad breath, itching, bleeding gums, etc.

\section{Differences between Western and Traditional Chinese} Medicine

The differences between diverse viewpoints and procedures within medicine can be illustrated by a tree figure (Figure 3). This example can also explain the functioning of the human body in a holistic view as affected by external factors (wind, cold, heat, dryness, humidity and diet) and internal factors (the emotions) (Figure 3). The tree has a trunk with several branches, where each branch represents each medical specialty and every single leaf that comes out of each branch represents the symptoms and diseases 
of every specialty. It is this visible part that is the object of study and practice in the western medical profession. This occurring due to the curriculum offered in the medical schools around the world, where the focus is to treat each leaf in every specialty (Figure 3).

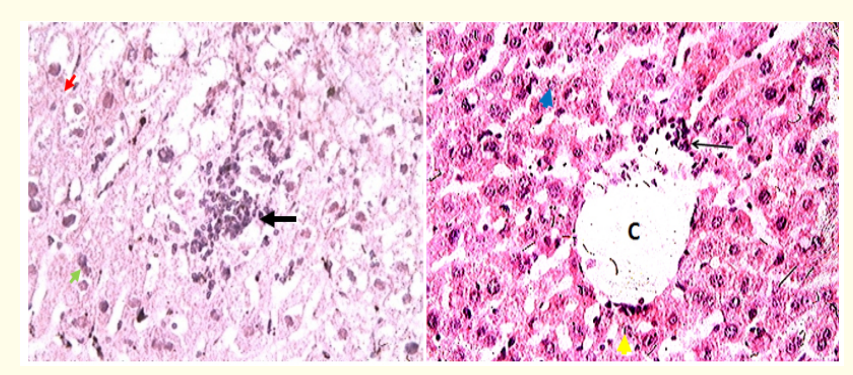

Figure 2

Figure 3

However, there are other points of view from other traditions. Such is the case with the Traditional Chinese Medicine. As we look at the figure, the part of the tree that is below the earth - invisible to the naked eye -, represents the roots of the tree. This part remains unknown to western medicine mainly because its existence is not recognized or discussed in the medical faculties (Figure 3).

Acupuncture, which is considered a medical speciality in Brazil since 1995 by the Federal Medical Council, is part of this Traditional Chinese Medicine. Some, but not all practitioners, literally go to their patients' root problems to understand the causes of the diseases and not just to recognize the symptoms. To understand these "roots" we turn toward the Theory of the Five Elements and the Yin/Yang Theory of Energy (Figure 6, 9).

The Qi energy concept is complex and deep and the whole system of Traditional Chinese Medicine, one of the oldest medical systems in the world, is entirely based on it. Differently from western medicine, which is based mainly on anatomy and physiology, in matter and its chemical reactions [5].

\section{Theory of the Five Elements}

I In the human body, each of these Five Elements represents an organ, and is responsible for an external sensorial organ. Each of these internal organs are the source of energy for their own functioning as well as their corresponding external sensorial organs and other functions. This system of energy is interlinked, and the influence of one element or one organ on the others is dynamic, and not fixed or isolated (Figure 4,6). The Wood element corresponds to the Liver, and has the eye as its external sensorial organ, being responsible for the vision. The Fire element corresponds to the Heart, and represents the tongue, and subsequently the language. The Earth element corresponds to the Spleen, representing the mouth, and is responsible for taste. The Air element corresponds to the Lungs, and represents the nose, and is responsible for the sense of smell. And the Water element corresponds to the Kidney, and represents the ears and is responsible for hearing. All this functioning is ruled by the flow of energy: Yin, Yang, Qi and Blood (Figure 4,5 )

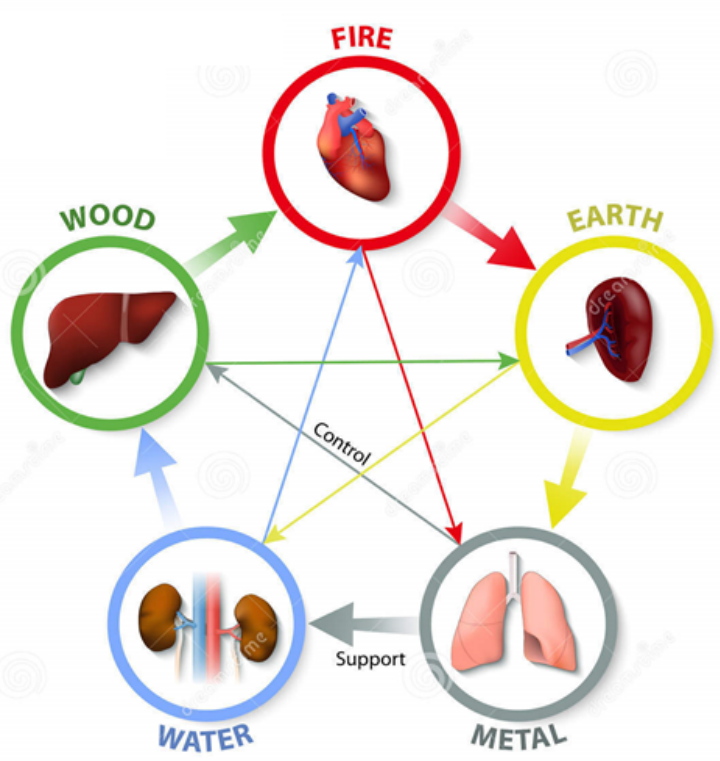

Figure 4

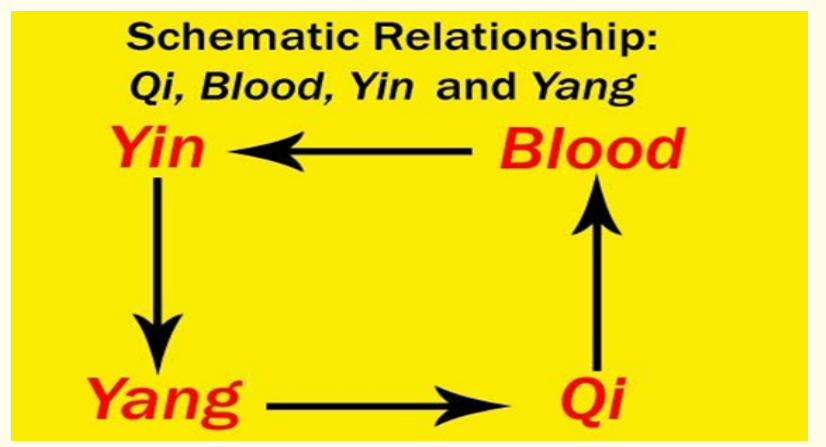

Figure 5

This theory was presented in 2015 at an Acupuncture Research Conference held at Harvard Medical School, in Boston, USA. The summary of this study says that if we treat these energy imbalances, we will treat all the patient's symptoms at the same time, even if the doctor does not know the patient has such a symptom. With this type of reasoning we will be able to treat all diseases of all specialties at the same time, as we treat in this case, the root of the problems and not only the symptoms (Figure 3,5).

Possible cause of patients still catching hospital infections despite prevention and control programs

One possible cause for patients still catch hospital infections despite prevention and control programs is due, personal opinion, to the model used by medicine itself today. This model was designed by Galen, a Greek physician, who lived 500 years after Hippocrates, and wrote theories about the functioning of the human body, which were implanted in almost all medical colleges of the West [8]. Possible cause of patients still catching hospital infections despite prevention and control programs His theories show the human being as divided into parts and each one is self sufficient and works separately from one another [8]. This great doctor was second only to the Greek, Hippocrates.

According to Hippocrates, diseases originate from the imbalance of the "four humours", which are blood, phlegm, yellow bile, and black bile. These humours determine the temperaments 
which are sanguine, phlegmatic, choleric and melancholic [9]. Every physical body has in itself the elements to recover, as nature itself is the only healer, and the physician simply limits himself to accompanying and obeying nature [10]. It can be compared to a car mechanic, if the patient is sick, his engine is out of tune, and our job is to get the engine tuned so that the patient has his or her functions working harmoniously, without having to use too many drugs. According to Hippocrates, everybody has in himself the elements to recover. It is only nature that heals, and the doctor should limit himself to accompany nature [10]. From the model presented by Galen, where each organ is isolated, working independently, it's difficult to see the human being as a whole. From the theory of the Five Elements in Traditional Chinese Medicine, all organs are interconnected with the flow of a self-generation and self-control system [4] (Figure 6). If an organ becomes ill, as in the case of the liver, which is directly affected by a diversity of drugs, this generates other disorders through the increase of internal Heat. As a consequence, this clinically mimics an infection such as reddening of the skin, surgical incision or purulent discharge. If we are not aware of what exactly is happening and treat this condition as an infection, a vicious cycle develops, worsening the patient's condition rather than improving it.

Then, from the point of view of Traditional Chinese Medicine, for instance a site with purulent hyperemia, secretion or catarrh, does not necessarily mean that the patient has a bacterial infection. It means that the patient has an imbalance of the internal energies and it is up to us, the physicians, to rebalance these energies so that the patient returns to his way of life without having to use many drugs. These drugs can worsen the vital energy [11], increasing the chance of "internal Heat", creating what clinically could be interpreted as infectious symptoms such as hyperemia, heat and reddening."

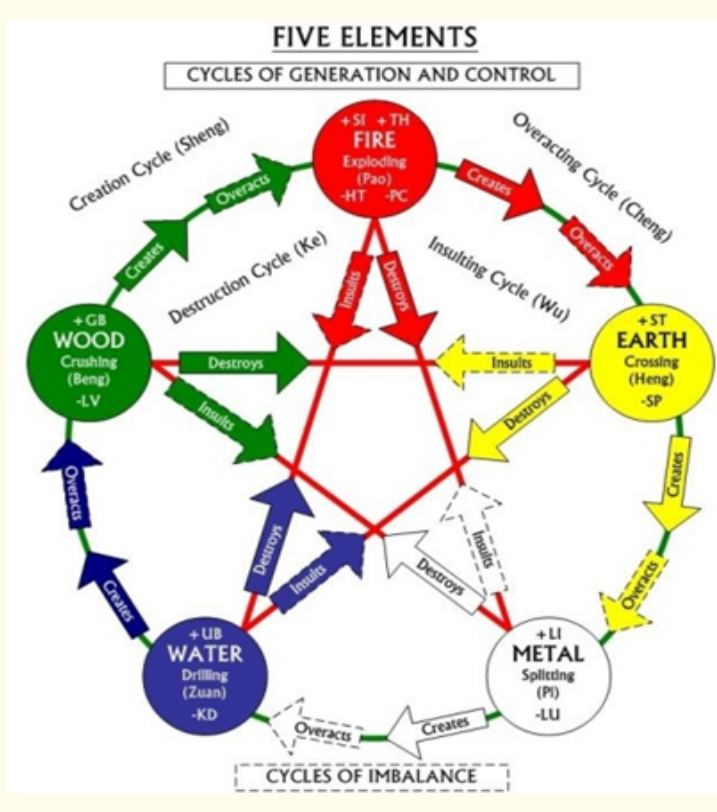

Figure 6

Then, from the point of view of Traditional Chinese Medicine, for instance a site with purulent hyperemia, secretion or catarrh, does not necessarily mean that the patient has a bacterial infection. It means that the patient has an imbalance of the internal energies and it is up to us, the physicians, to rebalance these energies so that the patient returns to his way of life without having to use many drugs. These drugs can worsen the vital energy [11], increasing the chance of "internal Heat", creating what clinically could be interpreted as infectious symptoms such as hyperemia, heat and reddening.

\section{Arndt-Schulz's Law}

The Arndt-Schulz's law, originally formulated in 1888 , helps to better understand the effects of different drug concentrations on an individual. The rule states that for all substances, small doses stimulate, moderate doses inhibit and overdoses kill [11]. According to this law, highly diluted drugs improve organic processes, while high concentrations harm health [11]. This hypothesis curiously coincides with homeopathic theory. Currently, Maturana and Varela, two Chilean researchers, have proved this and much regarding the sizes of doses in the role of stimulating or inhibiting the vitalities of living beings [11]."

It can be compare to a car mechanic, if the patient is sick, his engine is out of tune, and our job is to get the engine tuned so that the patient has his or her functions working harmoniously, without having to use too many drugs. According to Hippocrates, everybody has in himself the elements to recover. It is only nature that heals, and the doctor should limit himself to accompany nature [10].

\section{THE ARNDT=SCHULZ RULE OR LAW}

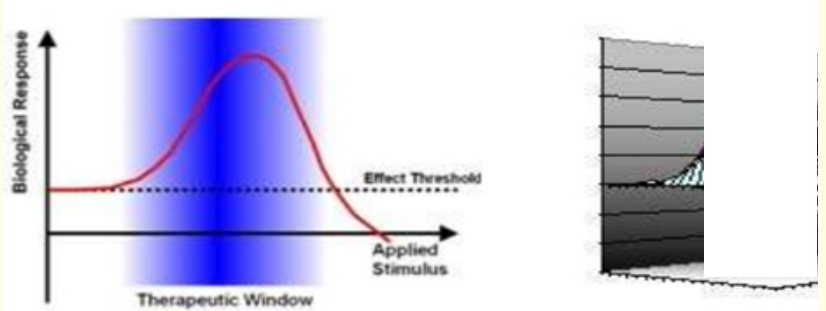

For every substance, small doses stimulate, moderate doses inhibit, large doses kill.

Figure 7

Water

In 2008, during a trip to China, there was interesting things to be noticed over the time spent on a one-month internship at a Beijing Hospital.

At the hotel room, there was a 20 liters' bottle of water with two little taps: one blue and one red. The blue one was for warm temperature water, and the red one was for boiling water to be prepared as tea or to drink.

What is the difference between drinking warm temperature water instead of drinking water at room temperature, or cold water?

The energy of the water is normally cold and if we drink this water constantly, we will weaken the energy of the Spleen and Pancreas, which are responsible for the absorption of nutrients, as well as maintaining the blood in the vessels [4]. This weakening could lead to Blood deficiency which in the Western tradition is diagnosed as anemia. However, this Blood deficiency could go undetected in laboratory exams showing normal blood count, yet the patient could be suffering from anemia. In relation to maintaining blood in the vessels, one good example is when a woman is menstruating for a whole month, which could mean Spleen and Pancreas deficiency.

Many of our hospitalized patients suffer from Spleen-Pancreas deficiency. Western customs of drinking cow's milk, and cold water or other cold drinks, and eating raw food, are a cause for this condition [6]. And this type of diet practiced in our hospitals, will continue the patients' Spleen-Pancreas deficiency, which will 
cause a liquid retention in their bodies, forming phlegm, catarrh, or mucus, increasing secretion in any wounds, they may have.

Normally in the hospital environment we can observe that patients usually receive cold water to drink, at least in Brazil and probably even in countries where the climate is much colder. The energy of water even at room temperature is cold and when ingested, it generates internal Heat, increasing the risk of reddening the skin, yellowing of secretion, etc. To avoid the production of internal Heat by drinking cold water or water at room temperature, it is better to drink warm $\left(37-55^{\circ} \mathrm{C} / 98.6-131^{\circ} \mathrm{F}\right)$ or hot $\left(55-75^{\circ} \mathrm{C} / 131^{\circ}\right.$ $-167^{\circ} \mathrm{F}$ ) water but not so hot as to cause sweating or to burn the tongue [12].

\section{Environment}

As far as external factors are concerned, what we should observe is the environment the patient is located at, and not only his apparent pathology. An understanding from the Traditional Chinese Medicine [4] and also from the importance Hippocrates gave to the environment and to inherited factors [9]. The external factors that may imbalance the patient's energy are: heat, humidity, dryness, cold and wind [4].

\section{Energy imbalance on admission}

Hospitalized patients can be divided in two groups, those who are chronically ill, having been medicated for some time and those who seem apparently healthy, having suffered an accident and were hospitalized, for example. In both cases there could be some degree of energy imbalance. This will depend on the energy of the parents when the patient was conceived and during gestation. After birth, the external factors such as wind, cold, heat humidity and dryness can also be factored in, together with the internal factors such as the emotions. Additionally, eating habits, chronic use of high levels of medication, lifestyle such as overwork, little leisure time, excessive sex, etc. could be the cause of this energy imbalance.

\section{Energy imbalances combination}

This figure shows twenty combinations of energy imbalances researched in 100 medical records selected out of 300 at an acupuncture clinic in Brazil (Figure 1.1). Each combination has its different symptoms or pathologies. In order of frequency they are as follows: Yin/Yang Deficiency (17\%); Yin/Yang/Blood Deficiency (13\%); Yang/Blood Deficiency (11\%); Blood Deficiency (1\%); Yin/ Yang/Blood Deficiency/Heat retention (5\%); Yin/Blood Deficiency (6\%); Yin/Yang/Qi Deficiency (9\%); Yin Deficiency (6\%); Yin/Qi Deficiency (5\%); Yin/Qi/Blood Deficiency (2\%); Yang/Blood Deficiency/Heat retention (4\%); Yin/Yang/Blood/Qi Deficiency (2\%); Blood Deficiency/Heat retention (1\%); Yin Deficiency/Heat retention (3\%); Yin/Yang/Blood/Qi Deficiency/Heat retention (6\%); Yin/Qi/Blood Deficiency/Heat retention (1\%); Yin/Yang/Qi Deficiency/Heat retention (4\%); Yin/Yang/Blood/Qi Deficiency (1\%); Yang Deficiency/Heat retention (1\%); Yin/Yang Deficiency/Heat retention (2\%).

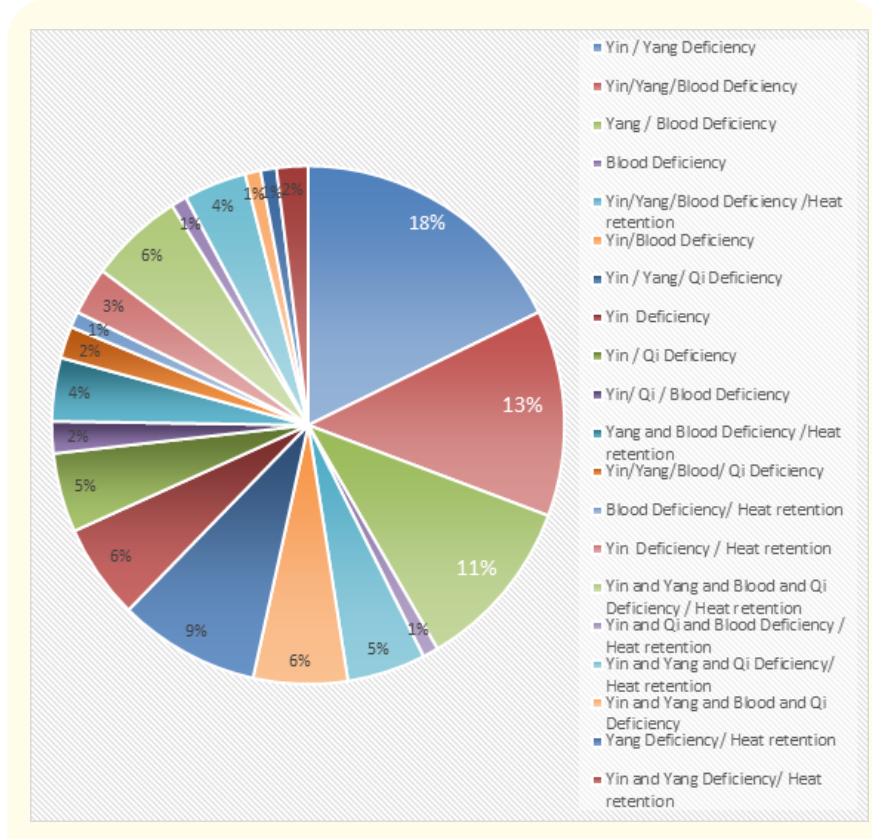

Figure 1.1

If the energy is low, it may or not form Heat. To evaluate this, a proof can be done through an anamnesis and/or physical examination [4]. Therefore, each patient may already at the moment of admittance to the hospital have some degree of energy imbalances, that are causing the patient's symptoms and disease but may be or not detected by the laboratory tests, depending on the degree and time of the evolution of these imbalances. So each patient could respond differently when subjected to the same type of treatment (Table 1).

\section{External factors \\ Cold and wind/Fan}

With regards to the external factors such as cold and/or wind, they can penetrate through the skin and internalize in an already weak patient with low energy, and cause a disease, such as a sore throat, sinusitis, pneumonia, diarrhea, etc. Therefore, a patient in a hospital environment should avoid cold and wind, because they can be the source of hospital infection, causing energy disturbances that penetrate the body and consequently cause heat formation, which simulates an infectious process. Definitely, fans should be avoided, not because it can be the source of bacteria and fungus but because they can induce a hospital infection by the wind itself.

\section{Cold and wind/air conditioning}

The same orientation should be used regarding the air conditioner, with cold and wind being the pathogenic factors [4]. They can generate hospital infection, because by penetrating the skin, they may internalize the patients' body, causing lack of appetite, coryza, pneumonia, intestinal constipation, body aches and more. These conditions are caused specifically by the energy imbalance and not by the infectious pathogens. If used in a hospital environment, airflow from any kind of fan or air conditioner should never be directed to a patient. 
Cover with sheet

We should always be careful to cover patients with at least one sheet, even in warm climates, so that the external pathogenic factor does not penetrate the skin. The sheet works to block this effect. Care must be taken to cover the upper part of the neck and thorax, because it is in these places that the external pathogenic factor begins to penetrate the body. The patient must be covered with warm blankets, mainly in less-developed countries where hospitals don't have central heating systems for the winter, to avoid the penetration of cold, which is the main cause to the beginning of the process of energy imbalance. This imbalance can clinically mimic an infectious process, generally interpreted as a hospital infection, and be treated with antibiotics. It complicates then the patient's condition, not only from the recognized side effects of these drugs, like destroying intestinal flora normal patient's protectors, as well as generating multi-resistant bacteria, worsening his energy imbalance, decreasing his vital energy and increasing the formation of internal heat that can also mimic an infectious process.

Bathe

This situation is very common in patients who bathe with an open window or an open door, causing a flow of air and wind in the bathroom. It weakens patients, being an initial factor in the imbalances that lead to a disease, simulating an infectious process, and thus causing a snowball effect. With the introduction of drugs, and often a broad spectrum of antibiotics, the energy condition may worsen [11], forming more Heat which could simulate an infection, as already explained above.

\section{Invasion of cold-fever}

The invasion of cold, inside the patient's body, can generate fever and chills. This symptom may be mistakenly interpreted as an infection, and empirical treatment with antibiotics is often initiated, resulting in a drop in vital energy [11], producing more Heat [4]. In some professional procedures, compresses of cold water or ice are usually recommended to reduce fever, leading to the entry of colder energy into the patient's body. Contrary to expected, it works counter wise the initial purpose as it was the cold the perpetrator of the fever provoking thus a "vicious cycle", and further complications to the patient.

\section{Use of medication}

Every procedure that generates increased use of medication can generate the reduction of vital energy [11] and consequently, the formation of Heat which causes signs and symptoms appearing to be infections. What in fact is happening here is that the resulting condition is actually internal energy disharmony. For example, it is very common to see black tea and coffee being served to patients after their dinner in hospitals, which is at least a common practice in Brazilian hospitals. This habit can cause an imbalance of inter- nal energy, often leading to insomnia in patients who are hospitalized, and who then reach for benzodiazepine, clonazepam or other kinds of medication to induce sleep. When taking this medication, according to the Law of Arndt and Shultz [11], a high concentration of the drug will cause a drop in vital energy. And according to Traditional Chinese Medicine, this drop in vital energy can cause the formation of Heat [4], or produce Heat from the direct aggression to the liver caused by the use of these kinds of medication [4].

\section{Diet}

A diet with what is regarded as a healthy program which recommends raw food [6] can be the cause of malabsorption of nutrients [4] which may be the reason for Spleen and Pancreas deficiency, leading to a possible anemia or deficiency of blood, and subsequently internal Heat formation.

\section{Water/enteral catheter}

Regarding the water that is used to wash the enteral catheter after feeding the patient, it is recommended it to be boiled [12]. Not only because of the risk of contaminants in the water, but to change the water energy from cold to neutral. Water needs to be warmed or boiled to a minimum of $37^{\circ} \mathrm{C}\left(98.6^{\circ} \mathrm{F}\right)$, and therefore in this way it cannot cause energy imbalances when entering the human body. Subsequently, Heat is not generated and the incidence of hospital infection is reduced

Within Traditional Chinese Medicine it is recommended that cow's milk and derivatives should be avoided. Enteral diets should not contain cow's milk [6], and they should be given always warm.

\section{Nebulization}

When it comes to nebulization, in addition to normal hygiene care, we must make sure the liquid to be inhaled is at body temperature, so as not to cause thermal shock as well as cough, asthma attack, fever, etc. and thus to be confused with an infectious process.

\section{Sign and symptoms of energy deficiency}

The signs and symptoms that show whether the patient is possibly deficient in energy, and with higher risks in contracting a nosocomial infection are:

1. Constipation - does not have a bowel movement every day (Blood deficiency)

2. Hot flashes (Yin deficiency)

3. Cold at the extremities (Yang deficiency)

4. Daytime sweating (Qi deficiency)

5. Night sweating (Yin deficiency)

6. Dry mouth (Heat Retention)

7. Bad breath (Heat Retention) 
8. Gingivitis and bleeding gums (Heat Retention)

9. Dermatitis on the skin with redness, pimples, etc... (Heat Retention)

10. Itchiness (Heat Retention)

11. Abdominal pain (Heat Retention)

12. Microscopic hematuria (Heat Retention)

\section{Apex ear bloodletting}

Here is shown the procedure used to take out the patient's Heat, using his ear lobe what is called bloodletting (Figure 8). Bloodletting is the blood withdrawal from a patient to cure or prevent illness and disease. Bloodletting was based on an ancient system of medicine in which blood and other bodily fluids were regarded as humors that had to remain in proper balance to maintain health [13].
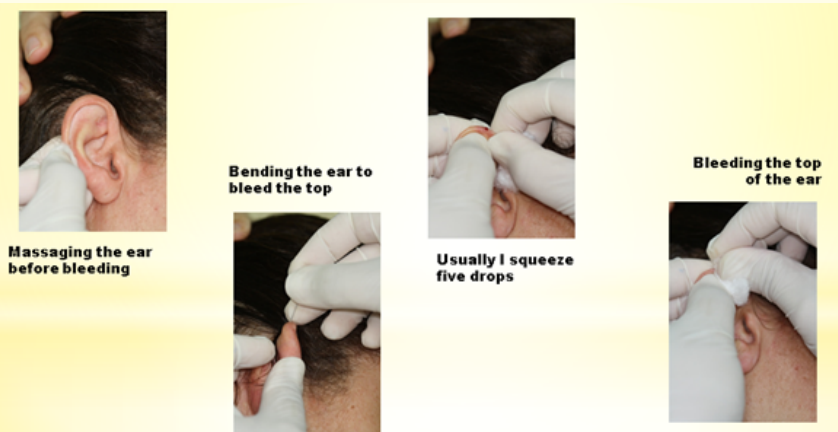

Figure 8

\section{Findings}

Having a whole, broader view of the patient (Yin, Yang, Qi, Blood energy and Heat retention), we can understand better the formation of hospital infection which is our body systemic energy reaction when undergoing normal hospital treatment.

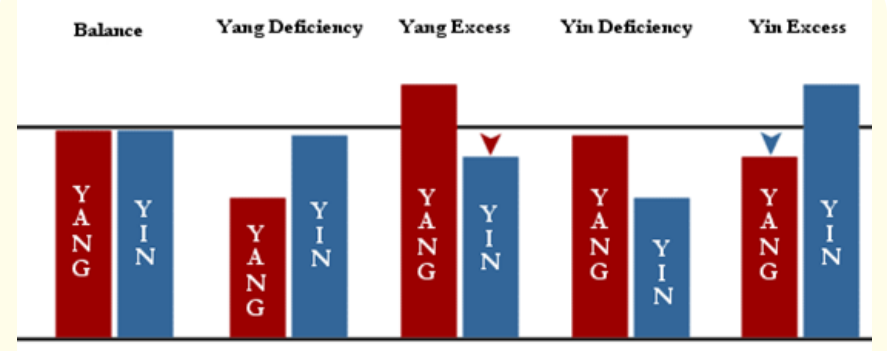

Figure 9

\section{Discussion}

Public health officials in the 1970s took notice of increasing numbers of HAIs, with their resultant increased morbidity, mortality, and hospital costs. Simultaneously, hospitals began implementing infection surveillance and control programs; however, their efficacy was unproven. In 1974, Haley and others at the CDC de- signed a nationwide study, the SENIC Project, to examine whether infection surveillance and control programs could lower the rates of HAIs. This study, performed over a 10-year period (1975 to 1985), examined HAI rates in a sampling of U.S. hospitals before and after the implementation of infection control programs. The SENIC study demonstrated that four components were essential to an effective infection prevention and control program. These included (i) surveillance with feedback of infection control rates to hospital staff, (ii) enforcement of preventative practices, (iii) a supervising IP to collect and analyze surveillance data, and (iv) the involvement of a physician or microbiologist with specialized training in infection prevention and control. Programs with these elements reduced rates of the four most common HAIs by $32 \%$ [1]

Hippocrates is traditionally considered the father of modern medicine, still influencing, 25 centuries after his time, various aspects of medical practice and ethics. His collected works include various references to infectious diseases that range from general observations on the nature of infection, hygiene, epidemiology, and the immune response, to detailed descriptions of syndromes such as tuberculous spondylitis, malaria, and tetanus [3]

Infectious diseases are prominent throughout the Hippocratic works; however, the Hippocratic approach, based on the general view of disease as a fluid dysregulation, means that most descriptions of clinical cases are vague and could be attributed to a wide variety of pathogens. Further confusion to the modern reader may emerge from the use in these works in terms such as cholera, leprosy, and typhus in a different manner: in fact, one of the typhus types described by Hippocrates represents the typical natural history of rheumatoid arthritis (43 ${ }^{\text {rd }}$ vignette of Internal affections). However, one cannot deny certain important aspects of the infectious diseases and immune responses recognized, such as the characterization of fever and swelling as part of the immune response, the seasonal emergence of various types of infection (hence the detailed description of climate evolution in the Epidemics books), the immune compromise related to excess alcohol intake, the relationship of certain diarrheal diseases to consumption of inadequately cooked meat and seafood, and foremost, the systematic approach to the progression of various clinical syndromes [3]

Associating disease to the body fluids disequilibrium may seem an ancient and outdated notion nowadays, but many of the clinical descriptions presented in the Corpus Hippocraticum (Hippocratic Collection) are still the archetypes of the natural history of certain infectious diseases and their collective interplay with the environment, climate, and society [3].

We can remember Hippocrates' words, that everyone has a doctor in him or her. We need to help this natural healing force within each one of us and acknowledge it to be the greatest force in getting well [8]. Let food be your medicine and medicine be your food [8].

Hippocrates developed a naturalistic approach to medicine, including the field of infectious diseases, introducing the concept of clinical observation to Western medicine [3]. 
Why do modern day researchers keep going back to Hippocrates? We are living in an era of rapid scientific advances in all aspects of infectious diseases, and it would seem reasonable that information drawn from ancient observations may not significantly alter our current perceptions. Yet, we keep returning: an ancient bug that has contributed to the shaping of history receives a certain respect; the disease is not something new but something that has been around for ages and should demand our attention [3].

TCM was deeply influenced by the ancient philosophical thoughts in the formation of its theoretical system, and a simple materialistic and dialectical thought goes through the whole theoretical system of TCM [4].

TCM stresses that body cannot be separated from the spirit. "Body" here refers to the human beings physical body, while "spirit" has many meanings, referring to (1) the functional manifestations of the changes and motions of the things in the world and their intrinsic laws; (2) the general outward manifestations of life activities, and (3) the mental activities of the human body. In accordance with TCM, the body is the basis to produce the spirit, while the spirit can regulate and control the activities of the body [4].

TCM seeks not only the causative factors of disease in nature, but also the intrinsic factors for the occurrence of disease in order to explain the pathologic changes of disease. Disease can be identified, prevented and treated. It suggests that preventive measures should be taken to prevent the occurrence of disease, and early diagnosis and treatment should be carried out once disease occurs [4].

TCM developed its unique theoretical system through a long clinical practice period. Compared with Western medicine, it has two basic features, the concept of holism and the Syndrome identification and corresponding treatment [4].

TCM places emphasis on the holism when analyzing pathological mechanism of disease, or the holistic reflection of a local disease, and the relations between the local disease and the systematic one. In other words, it stresses both the directly related organs or meridians of disease and the influence of these organs and meridians on the others. In the treatment of diseases, TCM also stresses that a proper therapeutic principle should be based on the concept of holism [4].

The theory of Yin/Yang and Five Elements has evolved into an important component part of TCM theory, and has exerted deep influence on the formation and development of the theoretical system of TCM [4].

The so-called "treating different disease with the same method" indicates that different diseases may be treated with the same method as a result of the same pathogenesis occurrence in a disease's development. From this we can see that disease treatment in TCM is based on different diseases' pathogenesis rather than the disease itself. This is what is called treating the same Syndrome with the same method and treating difference Syndrome with different methods [4].
Yin and Yang are a generalization of some related matter and phenomena, with opposite properties in nature. Two opposite aspects existing within one thing [4]. Cold or hot weather, upper or lower in locations, left or right, interior or exterior and motions or stillness. Two opposite forces of the nature which wane and wax alternately. All things contain Yin and Yang and Yin/Yang serves as the law of nature [4].

The material world is a unity resulting from the unity and opposite of Yin and Yang. Anything in the universe can be divided into the opposite Yin and Yang, cold or hot weather and the day and night [4].

Warm and excited the human body belongs to Yang; heaven and earth, the sun and the moon, water and fire, and male and female; is relative rather than absolute.

Yin and Yang may each be transformed in different conditions. Yin can transform into Yang and vice versa. There is still Yin and Yang within a Yin thing or a Yang thing. Day belongs to Yang and night to Yin, but comparatively, morning belongs to Yang within the Yang and afternoon belongs to Yin within the Yang, and compared with the first part of the night, the later part of the night belongs to Yang within the Yin and the first part belongs to Yin within the Yin [4].

Yin and Yang are the unity of the opposite. If this dynamic balance is disturbed, disaster in nature or disease in human body will follow. "Excess of Yin will lead to disorder of Yang while Excess of Yang will cause disorder of Yin". The opposite and restriction between Yin and Yang serve as an important factor preventing Excess of either of Yin and Yang and maintaining their balance [4].

The weather changes gradually from cold to warm and further to hot from the winter to the spring and from the spring to the summer, which is a process in which the Yin declines and Yang grows; while it changes gradually from hot to cool and further to cold from the summer to the autumn and from the autumn to the winter, which is a process in which Yang declines and Yin grows [4].

TCM holds that the human body is in an organic unity [4].

If the coordination between Yin and Yang are disturbed due to certain reasons, diseases will occur as a result of Excess or Deficiency of Yin or Yang [4].

TCM puts a high value on the prevention of disease. Theories on health preservation and prevention of disease are both based on the Yin/Yang theory [4].

\section{Conclusion}

In this study, we have attempted to explain why regardless the implanted infection control measures the patients still continue to contract nosocomial infections.

In order to control a hospital infection, understanding how the human body works in its deepest sense at the energy levels is very important. 
These laws include the theory of Five elements and Yin/Yang, and other theories that include both external and internal factors, dietary nutrition, to regulate their energy levels acting then in the reduction of hospital infection.

Looking at each symptom and understanding what each one represents at the body energy level, is a different approach from what most of us are doing now, and in this way, we would have the conditions to know how to treat the various forms of hospital infection, and also how to prevent their occurrence.

Each patient is unique, and each one has their own energy disturbances, and so they have to be evaluated individually, to have a successful result in their treatment. From this new point of view, associated with what we already have in our practice nowadays, this could be the beginning of a new perspective on hospital infection control. In helping to reduce infectious complications, costs, and the mortality rate caused by hospital infection and, more importantly, reducing the induction of bacterial resistance.

A broader view seems to show the necessity of seeing the patient as a whole; not only focusing on the disease in the prevention of these hospital infections.

\section{Bibliography}

1. Sydnor ERM and Perl TM. "Hospital Epidemiology and Infection Control in Acute-Care Settings". Clinical Microbiology Reviews 24.1 (2011): 141-173.

2. Mortell Manfred., et al. "Physician 'defiance" towards hand hygiene compliance: Is there a theory-practice-ethics gap?" Journal of the Saudi Heart Association 25.3 (2013): 203-208.

3. Pappas G., et al. "Insights into infectious disease in the era of Hippocrates". International Journal of Infectious Diseases 12.4 (2008): 347-350.

4. Bing Ouyang and Zhen Gu. "Essentials of Traditional Chinese Medicine". Translated by Lu Yubin. Beijing, China. Ed. Shandong Science and Technology Press (1996).

5. Dolic John. "What is Chi/Prana?" Journal of the American Medical Association 284.4 (2000).

6. Joerg Kastner. "Chinese Nutrition Therapy". Kastner, $2^{\text {nd }}$ Edition (1963).

7. Capra Fritjof. "O tao da física - Editora Cultrix". $2^{\text {nd }}$ edition (2013).

8. Fernandes Fernando. "Galeno e a universalização da medicina grego-romana” (2015).

9. Hipocrates.

10. Hippocrates.

11. Sharma Rajneesh Kumar. "Arndt Schultz Law and Its Applications".

12. Ling ML. "8 reasons to drink warm water" (2013)

13. Bloodletting

Volume 1 Issue 4 April 2018

(C) All rights are reserved by Huang Wei Ling. 